\title{
A CRITERION FOR THE NONPOROSITY OF A GENERAL CANTOR SET
}

\author{
PAUL D. HUMKE
}

(Communicated by R. Daniel Mauldin)

\begin{abstract}
The purpose of this paper is to prove that under quite general conditions, a closed nowhere dense perfect set is non- $\sigma$-porous if and only if it is nonporous.
\end{abstract}

This paper improves the results of the present author and B. S. Thomson [HT] and was written in response to a remark of L. Zaíček who pointed out that the proof in $[\mathrm{HT}]$ is incomplete. The notion of porosity was first introduced by E. P. Dolženko [D] in the course of a study of boundary behavior of certain complex-valued functions. Since that time it has been shown to play important and varied roles in the studies of quasiconformal mappings, functional analysis, and harmonic analysis, as well as in the area of classical real analysis with which the author is most familiar. Bibliographies of porosity results in real analysis can be found in the appendix to B. S. Thomson's survey [T1] or in L. Zajičceks survey $[\mathrm{Z} 2]$.

If $E$ is a subset of the real line $\mathbf{R}$, the porosity of $E$ at $x \in \mathbf{R}$ is defined as

$$
\limsup _{r \rightarrow 0} \frac{p(E, x, r)}{r},
$$

where $p(E, x, r)$ denotes the length of the longest interval in $(x-r, x+r) \cap$ $(\mathfrak{R} \backslash E)$. The set $E$ is porous if the porosity of $E$ at each of its points is positive, and strongly porous if the porosity of $E$ at each of its points is 1 . The set $E$ is called $\sigma$-porous ( $\sigma$-strongly porous) if it is the countable union of porous sets (strongly porous sets). An important and technically useful result about $\sigma$-porous sets was proved by L. Zajíček in [Z1].

Theorem Z. $A$ set $E$ is $\sigma$-porous iff $E=\bigcup_{n=1}^{\infty} E_{n}$, where each set $E_{n}$ has porosity at least $\frac{1}{2}$ at each of its points.

In order to establish our results, we first need some notation to describe perfect sets. Let $\Sigma_{n}$ denote the set of all binary sequences (0's and 1's) of length $n$, and let $\Sigma=\bigcup_{n=0}^{\infty} \Sigma_{n}$. If $\sigma \in \Sigma_{n}$, we let $|\sigma|=n$ and refer to $|\sigma|$

Received by the editors April 4, 1989; presented as part of a special session on real analysis at the Midwest Region Meeting of the American Mathematical Society, October 29, 1988.

1980 Mathematics Subject Classification (1985 Revision). Primary 26A03. 
as the height of $\sigma$. By $\sigma(k)$ we mean the $k$ th term of the sequence $\sigma$, and if $m<|\sigma|$, we let $\left.\sigma\right|_{m}$ denote $\sigma$ restricted to $\{1,2, \ldots, m\}$. If $\tau \in \Sigma_{n}$, we exhibit $\tau$ in expanded form as $\tau(1) \tau(2) \cdots \tau(n)$. We define an order $<$ on $\Sigma$ as follows:

First define $d: \Sigma \mapsto[0,1]$ by $d(\varnothing)=1 / 2$, and

$$
d(\sigma)=\frac{1}{2}+\sum_{n=1}^{|\sigma|} \frac{2 \sigma(n)-1}{2^{n+1}} \text { for } \sigma \neq \varnothing .
$$

Now, define $\sigma<\tau$ to mean $d(\sigma)<d(\tau)$. It is easy to see that $<$ is a total order on $\Sigma$ which is dense and unbordered.

Let $E$ be any set with a dense unbordered order. An order preserving surjection $g: \Sigma \mapsto E$ is said to induce a compatible binary labeling on $E$. Let $E$ be as above, and suppose that two functions $f_{i}: E \mapsto E(i=0,1)$ have been defined such that $f_{0 \sigma}(e)<e<f_{1 \sigma}(e)$ for each $\sigma \in \Sigma$, where $f_{\sigma}(e)=f_{\sigma(n)} \circ f_{\sigma(n-1)} \circ \cdots \circ f_{\sigma(1)}(e)$ for $\sigma \in \Sigma_{n}$. Fix $e \in E$ and define $g: E \mapsto E$ by $g(\sigma)=f_{\sigma}(e)$. Then $g$ induces a compatible binary labeling on $\left\{f_{\sigma}(e): \sigma \in \Sigma\right\} \subset E$.

In this paper we will be interested in sets of open intervals in $[0,1]$ whose natural order (that induced by $\mathbf{R}$ ) is dense and unbordered, and in compatible binary labelings of such sets. For notational convenience we will usually write $I_{\sigma}$ rather than $I(\sigma)$. As the order on the sets of intervals we consider is dense and unbordered, the intervals $I_{\sigma}$ are the intervals contiguous to a perfect set which we denote by $C\left(\left\{I_{\sigma}\right\}\right)$. The noncontiguous intervals of $C\left(\left\{I_{\sigma}\right\}\right)$ can then be labeled using $\Sigma$ as an index set in the obvious way; namely, $J_{\sigma}$ is that unique closed component of $[0,1] \backslash \bigcup_{|\tau|<|\sigma|} I_{\tau}$ which contains $I_{\sigma}$. It should be noted that although a given system $\left\{I_{\sigma}\right\}$ determines a unique perfect set, $C\left(\left\{I_{\sigma}\right\}\right)$, that perfect set will also be determined by any other system which is an appropriate relabeling of $\left\{I_{\sigma}\right\}$. Here we will consider systems which are centered in the sense that

$$
2 \gamma \equiv \liminf _{|\sigma| \rightarrow \infty}\left\{\frac{\left|J_{\sigma i}\right|}{\left|J_{\sigma}\right|}: i=0,1\right\} \neq 0 .
$$

Suppose $s: \Sigma \mapsto \mathbf{R}_{+}$is such that

$$
0<s(\sigma)<\min \left\{\left|J_{\sigma 100}\right|,\left|J_{\sigma 011}\right|\right\} \quad \text { for each } \sigma \in \Sigma .
$$

Define

1. $k_{1}^{1}(\sigma)=\tau=\sigma 100 \ldots 0$ such that $\left|J_{\tau 0}\right|<s(\sigma) \leq\left|J_{\tau}\right|$.

2. $k_{1}^{n}(\sigma)=k_{1}^{1}\left(k_{1}^{n-1}(\sigma)\right)$ if $n>1$.

The functions $k_{0}^{n}(\sigma)$ are defined similarly with the roles of 0 and 1 interchanged. Finally, define $\tilde{I}_{\sigma}=I_{\sigma} \cup R_{\sigma} \cup L_{\sigma}$ where

$$
R_{\sigma}=\left[\bigcup_{n=1}^{\infty} J_{k_{1}^{n}(\sigma) 0}\right] \cup\left[\bigcup_{n=1}^{\infty} I_{k_{1}^{n}(\sigma)}\right] \text { and } L_{\sigma}=\left[\bigcup_{n=1}^{\infty} J_{k_{0}^{n}(\sigma) 1}\right] \cup\left[\bigcup_{n=1}^{\infty} I_{k_{0}^{n}(\sigma)}\right] \text {. }
$$


Lemma 1. Let $\left\{I_{\sigma}\right\}$ be centered and s satisfy (2). Then if $\left|\sigma_{1}\right|>\left|\sigma_{2}\right|$ and $\tilde{I}_{\sigma_{1}} \cap \tilde{I}_{\sigma_{2}} \neq \varnothing$, we have $\tilde{I}_{\sigma_{1}} \subseteq \tilde{I}_{\sigma_{2}}$.

Proof. Suppose $\left|\sigma_{1}\right|>\left|\sigma_{2}\right|$ and $\tilde{I}_{\sigma_{1}} \cap \tilde{I}_{\sigma_{2}} \neq \varnothing$. Then either $R_{\sigma_{2}} \cap L_{\sigma_{1}} \neq \varnothing$ or $R_{\sigma_{1}} \cap L_{\sigma_{2}} \neq \varnothing$. Suppose the latter and let $n$ be the least index such that $J_{k_{1}^{n}\left(\sigma_{1}\right) 0} \cap L_{\sigma_{2}} \neq \varnothing$. Let $m$ be the least index such that $J_{k_{1}^{n}\left(\sigma_{1}\right) 0} \cap J_{k_{0}^{m}\left(\sigma_{2}\right) 1} \neq \varnothing$. As these are two of the noncontiguous intervals of a Cantor set, either $J_{k-1^{n}\left(\sigma_{1}\right) 0} \subseteq$ $J_{k_{0}^{m}\left(\sigma_{2}\right) 1}$ or $J_{k_{0}^{m}\left(\sigma_{2}\right) 1} \subseteq J_{k_{1}^{n}\left(\sigma_{1}\right) 0}$. It follows from the minimality of $n$ that the former is not possible unless $n=1$. But in this case, $\sigma_{1}=k_{0}^{m}\left(\sigma_{2}\right)$ contradicting the fact that $\left|\sigma_{1}\right|>\left|\sigma_{2}\right|$. We now turn to the second case. If $J_{k_{0}^{m}\left(\sigma_{2}\right) 1}$ does not share a right endpoint with $J_{k_{1}^{n}\left(\sigma_{1}\right) 0}$, then as $m$ is minimal, $I_{\sigma_{2}} \subseteq J_{k_{1}^{n}\left(\sigma_{1}\right) 0}$. This implies that $\tilde{I}_{\sigma_{2}} \subseteq J_{k_{1}^{n}\left(\sigma_{1}\right) 0}$ and we are finished. If $J_{k_{0}^{m}\left(\sigma_{2}\right) 1}$ shares a right endpoint with $J_{k_{1}^{n}\left(\sigma_{1}\right) 0}$, then $I_{k_{1}^{n}\left(\sigma_{1}\right)}=I_{k_{0}^{m-1}\left(\sigma_{2}\right)}$ and so $k_{1}^{n}\left(\sigma_{1}\right)=k_{0}^{m-1}\left(\sigma_{2}\right)$. This, however, is impossible if $m>1$ because $k_{1}^{n}\left(\sigma_{1}\right)$ ends in 0 while $k_{0}^{m-1}\left(\sigma_{2}\right)$ ends in 1. If $m=1$, then $k_{1}^{n}\left(\sigma_{1}\right)=\sigma_{2}$, in which case $R_{\sigma_{2}}=R_{k_{1}^{n}\left(\sigma_{1}\right)} \subseteq R_{\sigma_{1}}$. But then $I_{\sigma_{1}} \cup L_{\sigma_{2}} \subseteq J_{k_{1}^{n}\left(\sigma_{1}\right) 0}$ and the result follows.

Lemma 2. If $\left\{I_{\sigma}\right\}$ is centered and s satisfies condition (2), then for every $\sigma$ with $|\sigma|$ sufficiently large $\gamma\left|J_{k_{i}^{1}(\sigma)}\right|<s(\sigma) \leq\left|J_{k_{i}^{1}(\sigma)}\right|, i=0,1$.

Proof. If $\sigma$ is chosen so large that $\left|J_{\sigma i}\right| /\left|J_{\sigma}\right|>\gamma$, then the proof follows easily from (2) and the definitions of $k_{i}^{1}, i=0,1$.

Lemma 3. Suppose $\left\{I_{\sigma}\right\}$ is centered and satisfies (2). Then $\tilde{I}_{\sigma} \subseteq J_{k_{0}^{1}(\sigma)} \cup$ $J_{k_{1}^{1}(\sigma)} \cup I_{\sigma}$ for all $\sigma \in \Sigma$.

Proof. We prove that $R_{\sigma} \subset J_{k_{1}^{1}(\sigma)}$ and $L_{\sigma} \subset J_{k_{0}^{1}(\sigma)}$. To prove the former it suffices to show that $J_{k_{1}^{n}(\sigma)} \subset J_{k_{1}^{1}(\sigma)}$ for all $n \in \mathbf{N}$. This is certainly true for $n=1$. Assuming it is true for $n$, we consider

$$
J_{k_{1}^{n+1}(\sigma)}=J_{k_{1}^{1}\left(k_{1}^{n}(\sigma)\right)} \subset J_{k_{1}^{n}(\sigma) 1} \subset J_{k_{1}^{n}(\sigma)} \subset J_{k_{1}^{1}(\sigma)^{\prime}} .
$$

This completes the proof of the first part; the proof of the second part is similar.

The intervals $\left\{\tilde{I}_{\sigma}\right\}$ again determine a Cantor set and, as we will investigate inductively, we wish to show that this new Cantor set can be described by a centered sequence of intervals. In the remarks which follow, we identify that centered sequence and the appropriate binary labeling which specifies its order. We define $\sigma_{1} \sim \sigma_{2}$ if there exists a $\sigma$ such that $\tilde{I}_{\sigma_{1}} \cup \tilde{I}_{\sigma_{2}} \subset \tilde{I}_{\sigma}$. It follows from Lemma 1 that $\sim$ is an equivalence relation and we denote the equivalence class of $\sigma$ by $\tilde{\sigma}$. The necessarily unique element of $\tilde{\sigma}$ of least height is denoted by $\sigma^{*}$. If $\sigma \in \Sigma$, we let

$$
\sigma_{-}=\{\sigma\} \cup\{\sigma 0 \tau: \tau \in \Sigma\} \quad \text { and } \quad \sigma_{+}=\{\sigma\} \cup\{\sigma 1 \tau: \tau \in \Sigma\} \text {. }
$$

It then follows that

$$
\tilde{\sigma}=\left\{\sigma^{*}\right\} \cup\left[\bigcup_{n=1}^{\infty}\left(k_{1}^{n}\left(\sigma^{*}\right)\right)_{-}\right] \cup\left[\bigcup_{n=1}^{\infty}\left(k_{0}^{n}\left(\sigma^{*}\right)\right)_{+}\right] .
$$


Now, let $E=\left\{\tilde{I}_{\sigma^{*}}: \sigma \in \Sigma\right\}$ and define $f_{i}: E \rightarrow E, i=0,1$, as follows:

1. $f_{0}\left(\tilde{I}_{\varnothing}\right)=\tilde{I}_{0}$ and $f_{1}\left(\tilde{I}_{\varnothing}\right)=\tilde{I}_{1}$.

2. Suppose that $f_{0}\left(\tilde{I}_{\sigma^{*}}\right)$ and $f_{1}\left(\tilde{I}_{\sigma^{*}}\right)$ have been defined for all $\sigma \in \bigcup_{k=0}^{n-1} \Sigma_{k}$ and let $\sigma \in \Sigma_{n}$. If there is a $\tau \in \bigcup_{k=0}^{n-1} \Sigma_{k}$ such that $\sigma^{*}=\tau$, then $f_{0}\left(\tilde{I}_{\sigma^{*}}\right)$ and $f_{1}\left(\tilde{I}_{\sigma^{*}}\right)$ have already been defined. Suppose, then, that $\sigma=\sigma^{*}$, and let $\tau \equiv \sigma 0$. If $\tau=\tau^{*}$, define $f_{0}\left(\tilde{I}_{\sigma^{*}}\right)=\tilde{I}_{\tau}$. If, however, $\tau \neq \tau^{*}$, then either

$$
\tau=\bigcup_{n=1}^{\infty}\left(k_{1}^{n}\left(\tau^{*}\right)\right)_{-} \quad \text { or } \quad \tau \in \bigcup_{n=1}^{\infty}\left(k_{0}^{n}\left(\tau^{*}\right)\right)_{+} .
$$

In the latter case there is a unique $m$ such that $\tau \in\left(k_{0}^{m}\left(\tau^{*}\right)\right)_{+}$. That is, $\tau=k_{0}^{m}\left(\tau^{*}\right) 1 \tau_{1}$ for some $\tau_{1} \in \Sigma$. From this it is easy to see that $\sigma \in\left(k_{0}^{m}\left(\tau^{*}\right)\right)_{+}$, and as $\sigma=\sigma^{*}$, this case is impossible. In the former case there is a unique $m$ such that $\tau \in\left(k_{1}^{m}\left(\tau^{*}\right)\right)_{-}$. Using the fact that $\tau \not \nsim \sigma$ we conclude that $\tau=k_{1}^{m}\left(\tau^{*}\right)$. It now follows from Lemma 5 that $\sigma 01^{*}=\sigma 01$, and we define $f_{0}\left(\tilde{I}_{\sigma^{*}}\right)=\tilde{I}_{\sigma 01}$. The function $f_{1}$ is defined similarly. Using the remarks at the beginning of the paper, and assuming the verity of Lemma 5 , there is a compatible binary ordering $g: \Sigma \rightarrow E$. Furthermore, if $\sigma=\sigma^{*}$ and $g(\tau)=\tilde{I}_{\sigma}$, then $g(\tau i)=\tilde{I}_{f_{i}(\sigma)}$ for $i=0,1$. We denote by $\tilde{J}_{\sigma}$ the unique closed component of $[0,1] \backslash \bigcup_{|\zeta|<|\tau|} g(\zeta)$ which contains $g(\tau)=\tilde{I}_{\sigma}$. In the future when we refer to $\tilde{I}_{\sigma}$ or $\tilde{J}_{\sigma}$ we will implicitly assume that $\sigma=\sigma *$. The existence of a compatible binary labeling is then established with the proof of Lemma 5. Diagram 1 illustrates $\tilde{\sigma}$.

Lemma 5. Suppose $\sigma \in \Sigma$ with $\sigma=\sigma^{*}$, and let $\tau=k_{i}^{n}(\sigma)$ for some $n \in \mathbf{N}$, $i=0,1$. Then $\tau i=\tau i^{*}$.

Proof. Suppose $\tau 1^{*} \equiv \mu \neq \tau 1$. Then

$$
\tau 1 \in\left[\bigcup_{n=1}^{\infty}\left(k_{1}^{n}(\mu)\right)_{-}\right] \cup\left[\bigcup_{n=1}^{\infty}\left(k_{0}^{n}(\mu)\right)_{+}\right] .
$$

Case 1. If $\tau 1 \in\left(k_{1}^{m}(\mu)\right)_{-}$for some $m$, then $\tau 1=k_{1}^{m}(\mu) 0 \nu 1$ for some $\nu \in \Sigma$. Hence, $k_{1}^{n}(\sigma) \equiv \tau=k_{1}^{m}(\mu) 0 \nu$ so that $\sigma \sim \mu$. From this it follows that $\tau 1 \sim \sigma$ since $\tau 1 \sim \mu$, but this is impossible.

Case 2. If $\tau 1 \in\left(k_{0}^{n}(\mu)\right)_{+}$for some $m$, then $\tau 1=k_{0}^{m}(\mu) 1 \nu$ for some $\nu \in \Sigma$. If $\nu \neq \varnothing$, then $\sigma \sim \mu$ which, as in Case 1, is not possible. If $\nu=\varnothing$, however, then $k_{1}^{n}(\sigma)=k_{0}^{m}(\mu)$, and this is also not possible.

Hence, $\tau 1^{*}=\tau 1$ and the lemma is proved.

Lemma 6. Let $\left\{I_{\sigma}\right\}$ be centered, $s$ satisfy condition (2), and $\sigma=\sigma^{*}$. Then whenever $|\sigma|$ is sufficiently large, we obtain

$$
\left|J_{\sigma 0}\right| \geq\left|\tilde{J}_{f_{i}(\sigma)}\right| \geq\left|J_{\sigma 01}\right|-\frac{s(\sigma)+s(\sigma 0)}{\gamma}, \quad \text { for } i=0,1 .
$$




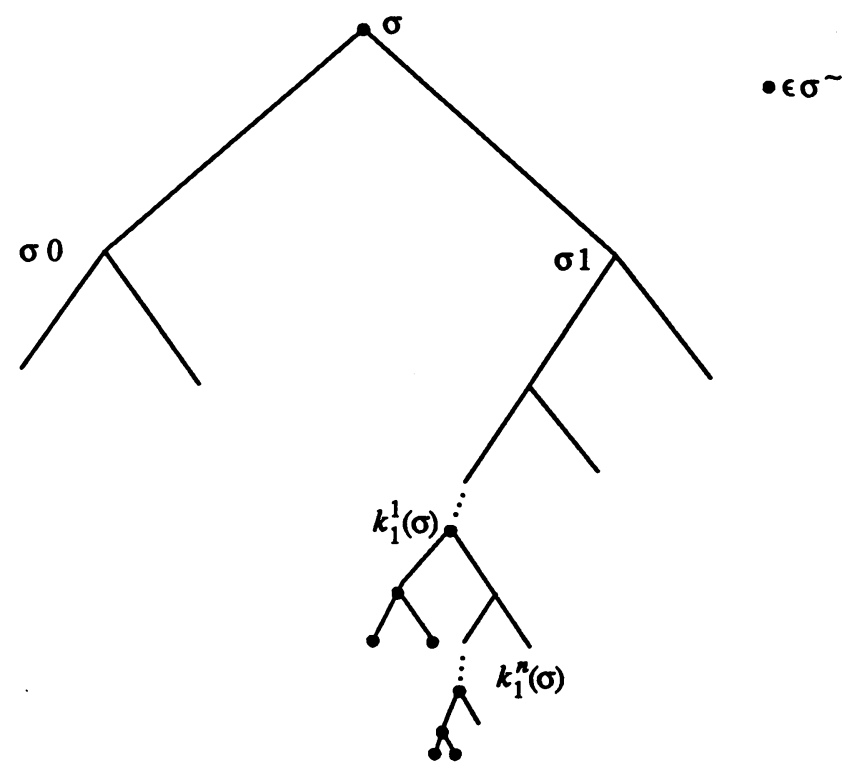

DiAgram 1

Proof.

Case 1. Suppose $f_{0}(\sigma)=\sigma 01$

$$
\begin{aligned}
\left|J_{\sigma 0}\right| & \geq\left|\tilde{J}_{f_{0}(\sigma)}\right|=\left|J_{\sigma 01}\right|-\left|R_{\sigma 0}\right|-\left|L_{\sigma}\right| \\
& \geq\left|J_{\sigma 01}\right|-\left|J_{k_{1}^{1}(\sigma 0)}\right|-\left|J_{k_{1}^{1}(\sigma)}\right| \geq\left|J_{\sigma 01}\right|-\frac{s(\sigma)+s(\sigma 0)}{\gamma} .
\end{aligned}
$$

Case 2. Suppose $f_{0}(\sigma)=\sigma 0$. Then there is a $\tau$ with $|\tau|<|\sigma|$ such that

$$
\begin{aligned}
\left|J_{\sigma 0}\right| & \geq\left|\tilde{J}_{f_{0}(\sigma)}\right|=\left|J_{\sigma 0}\right|-\left|R_{\tau}\right|-\left|L_{\sigma}\right| \\
& \geq\left|J_{\sigma 0}\right|-\left|J_{k_{1}^{1}(\tau)}\right|-\left|J_{k_{1}^{1}(\sigma)}\right| \geq\left|J_{\sigma 01}\right|-\left|J_{k_{1}^{1}(\sigma)}\right| \\
& \geq\left|J_{\sigma 01}\right|-\frac{s(\sigma)}{\gamma} .
\end{aligned}
$$

Lemma 7. Suppose $\left\{I_{\sigma}\right\}$ is centered and s satisfies condition (2). Suppose, too, that

$$
\limsup _{|\sigma| \rightarrow \infty} \frac{s(\sigma)}{\left|I_{\sigma}\right|}<\gamma^{3}
$$

Then $\left\{\tilde{I}_{\sigma}\right\}$ is centered.

Proof. Note that the condition $s(\sigma)<\gamma^{3}\left|I_{\sigma}\right|$ implies condition (2). In order to invoke our results on equivalence classes, however, condition (2) must always 
be satisfied. For $|\sigma|$ sufficiently large,

$$
\begin{aligned}
\frac{\left|\tilde{J}_{f_{0}(\sigma)}\right|}{\left|\tilde{J}_{\sigma}\right|} & \geq \frac{\left|J_{\sigma 0}\right|-\frac{s(\sigma)+s(\sigma 0)}{\gamma}}{\left|J_{\sigma}\right|} \\
& \geq \frac{\left|J_{\sigma 0}\right|-\gamma^{2}\left(\left|I_{\sigma}\right|+\left|I_{\sigma 0}\right|\right)}{\left|J_{\sigma}\right|} \geq \frac{\left|J_{\sigma 0}\right|}{\left|J_{\sigma}\right|}-2 \gamma^{2} .
\end{aligned}
$$

The liminf of this last quantity exceeds $\gamma-2 \gamma^{2}$ which, as $\gamma<\frac{1}{4}$, is positive.

Theorem 1. Let $\left\{I_{\sigma}\right\}$ be centered, $s$ satisfy condition '2), and

$$
0<\gamma^{*}<\liminf _{|\sigma| \rightarrow \infty}\left\{\frac{\left|J_{\sigma i}\right|}{\left|J_{\sigma}\right|}: i=0,1\right\} .
$$

Suppose, too, that $\lim _{|\sigma| \rightarrow \infty}\left|I_{\sigma}\right| /\left|J_{\sigma}\right|=0$, and that there is an $N \in \mathbf{N}$ such that

$$
\limsup _{|\sigma| \rightarrow \infty} \frac{s(\sigma)}{\left|I_{\sigma}\right|} \leq N \text {. }
$$

Then

1. $\left\{\tilde{I}_{\sigma}\right\}$ is centered with $\gamma^{*}<\liminf _{|\sigma| \rightarrow \infty}\left\{\left|\tilde{J}_{f_{i}(\sigma)}\right| /\left|\tilde{J}_{\sigma}\right|: i=0,1\right\}$, and

2. $\lim _{|\sigma| \rightarrow \infty}\left|\tilde{I}_{\sigma}\right| /\left|\tilde{J}_{\sigma}\right|=0$,

Proof.

$$
\begin{aligned}
\frac{\left|\tilde{J}_{f_{0}(\sigma)}\right|}{\left|\tilde{J}_{\sigma}\right|} & \geq \frac{\left|J_{\sigma 0}\right|-\frac{s(\sigma)+s(\sigma 0)}{\gamma}}{\left|J_{\sigma}\right|} \\
& \geq \frac{\left|J_{\sigma 0}\right|-\frac{N}{\gamma}\left(\left|I_{\sigma}\right|+\left|I_{\sigma 0}\right|\right)}{\left|J_{\sigma}\right|} \\
& =\frac{\left|J_{\sigma 0}\right|}{\left|J_{\sigma}\right|}-\frac{N}{\gamma}\left[\frac{\left|I_{0}\right|}{\left|J_{\sigma}\right|}+\frac{\left|I_{\sigma 0}\right|}{\left|J_{\sigma 0}\right|} \mid \frac{J_{\sigma 0} \mid}{\left|J_{\sigma}\right|}\right] .
\end{aligned}
$$

The first conclusion now follows by taking the liminf of both sides of the preceding equation. For the second, consider

$$
\begin{aligned}
& \frac{\left|\tilde{I}_{\sigma}\right|}{\left|\tilde{J}_{\sigma}\right|} \leq \frac{\left|I_{\sigma}\right|+\left|J_{k_{1}^{1}(\sigma)}\right|+\left|J_{k_{0}^{1}(\sigma)}\right|}{\left|J_{\sigma 0}\right|-\frac{s(\sigma)+s(\sigma 0)}{\gamma}} \\
& \leq \frac{\left|I_{\sigma}\right|+\frac{2 s(\sigma)}{\gamma}}{\left|J_{\sigma 0}\right|-\frac{s(\sigma)+s(\sigma 0)}{\gamma}}<\frac{\left|I_{\sigma}\right|+\frac{2 N}{\gamma}\left|I_{\sigma}\right|}{\left|J_{\sigma 0}\right|-\frac{N}{\gamma}\left(\left|I_{\sigma}\right|+\left|I_{\sigma 0}\right|\right)} \\
& =\frac{\left|I_{\sigma}\right|}{\left|J_{\sigma}\right|}\left[\frac{1+\frac{2 N}{\gamma}}{\frac{\left|J_{\sigma 0}\right|}{J_{\sigma} \mid}-\frac{N}{\gamma}\left(\frac{\left|I_{\sigma}\right|}{\left|J_{\sigma}\right|}+\frac{\left|I_{\sigma 0}\right|}{\left|J_{\sigma 0}\right|} \mid \frac{J_{\sigma 0} \mid}{J_{\sigma} \mid}\right)}\right] \text {. }
\end{aligned}
$$

From this it is easy to see that the second conclusion holds.

Theorem 2. Suppose that $\left\{I_{\sigma}\right\}$ is centered and $\lim _{|\sigma| \rightarrow \infty}\left|I_{\sigma}\right| /\left|J_{\sigma}\right|=0$. Then $C\left(\left\{I_{\sigma}\right\}\right)$ is not $\sigma$-porous. 
Proof. Suppose $C \equiv C\left(\left\{I_{\sigma}\right\}\right)$ is $\sigma$-porous. Then it follows from Theorem $\mathrm{Z}$ that $C=\bigcup_{n=1}^{\infty} E_{n}$ where $p\left(E_{n}, x\right) \geq \frac{1}{2}$ for each $x \in E_{n}$. Following Zajiček's technique, we construct a nested sequence of closed subsets of $C$ as follows:

1A. If $E_{1}$ is dense in $C$, we choose a function $s(\sigma)$ satisfying condition (2) such that

$$
\lim _{|\sigma| \rightarrow \infty} \frac{s(\sigma)}{\left|I_{\sigma}\right|}=\frac{1}{\gamma}
$$

It follows from Theorem 1 that $\left\{\tilde{I}_{\sigma}\right\}$ satisfies the hypothesis of Theorem 2, and we let $F_{1}=C\left(\left\{\tilde{I}_{\sigma}\right\}\right)$.

1B. If $E_{1}$ misses a portion of $C$, we let $F_{1}$ be one such portion of $C$ missing $E_{1}$.

In either case, $F_{1} \cap E_{1}=\varnothing$ and $F_{1}$ is determined by a centered system of intervals, which we denote by $\left\{I_{\sigma}^{1}\right\}$. Now suppose that $F_{n}$ has been defined such that $F_{n} \cap E_{n}=\varnothing, F_{n} \subset F_{n-1}$, and $F_{n}$ is determined by a centered system of intervals, $\left\{I_{\sigma}^{n}\right\}$.

$(n+1) \mathrm{A}$. If $E_{n+1}$ is dense in $F_{n}$, we choose a function $s(\sigma)$ satisfying condition (2) such that

$$
\lim _{|\sigma| \rightarrow \infty} \frac{s(\sigma)}{\left|I_{\sigma}^{n}\right|}=\frac{1}{\gamma}
$$

As in 1A, it follows from Theorem 1 that $\left\{\tilde{I}_{\sigma}^{n}\right\}$ satisfies the hypothesis of Theorem 2, and we let $F_{n+1}=C\left(\left\{\tilde{I}_{\sigma}^{n}\right\}\right)$.

$(n+1)$ B. If $E_{n+1}$ misses a portion of $F_{n}$, we define $F_{n+1}$ to be that portion of $F_{n}$ which misses $E_{n+1}$.

Again, in either case, $F_{n+1} \cap E_{n+1}=\varnothing, F_{n+1} \subset F_{n}$, and $F_{n+1}$ is determined by a centered system of intervals.

It is evident, however, that $\bigcup_{n=1}^{\infty} E_{n}$ misses $\bigcap_{n=1}^{\infty} F_{n}$ and this contradiction completes the proof.

Theorem 3. Suppose that $\left\{I_{\sigma}\right\}$ is centered. Then $C\left(\left\{I_{\sigma}\right\}\right)$ is not $\sigma$-strongly porous.

Proof. This proof proceeds as in Theorem 2 with the exception that Lemma 7 is invoked in steps A rather than Theorem 1.

If $\zeta_{n}<\frac{1}{2}$ for all $N \in \mathbf{N}$, then the sequence $\left\{\zeta_{n}\right\}$ can be used to define a symmetric perfect subset, $C\left(\left\{\zeta_{n}\right\}\right)$ of $[0,1]$. See [T2] or [Z2] for details. The following theorem can be found in [T2].

Theorem. If $C\left(\left\{\zeta_{n}\right\}\right) \equiv C$ is the symmetric subset of $[0,1]$ determined by the sequence $\left\{\zeta_{n}\right\}$, then

(1) $\left\{1-2 \zeta_{n}\right\}$ is summable if and only if $C$ is not measure zero.

(2) If $\lim \zeta_{n}=\frac{1}{2}$, then $C$ is not $\sigma$-porous.

(3) If $\liminf \zeta_{n}<\frac{1}{2}$, then $C$ is porous. 
(4) If $\liminf \zeta_{n}=0$, then $C$ is strongly porous.

(5) If $\liminf \zeta_{n}>0$, then $C$ is not $\sigma$-strongly porous.

The difficult parts of this theorem, parts 2 and 5, follow directly from Theorems 2 and 3 of this paper. A rather complete survey of porosity and $\sigma$-porosity can be found in $[\mathrm{Z} 2]$.

\section{REFERENCES}

[D] E. P. Dolženko, Boundary properties of arbitrary functions, Izv. Akad. Nauk SSSR Ser. Mat. 31 (1967), 3-14. (Russian)

[HT] P. D. Humke and B. S. Thomson, A porosity characterization of symmetric perfect sets, Classical Real Analysis, Contemp. Math., no. 42, Amer. Math. Soc., Providence, RI, 1985, pp. 81-85.

[T1] B. S. Thomson, Derivation basis on the real line. II, Real Anal. Exchange, vol. 8, no. 2, 1982/83, pp. 278-442.

[T2] _ Real functions, Lecture Notes in Math., vol. 1170, Springer-Verlag, Berlin and New York, 1985.

[Z1] L. Zajiček, Sets of $\sigma$-porosity and sets of $\sigma$-porosity (q), Časopis Pěst. Mat. 101 (1976), 350-359.

[Z2] _-, Porosity and $\sigma$-porosity, Real Anal. Exchange, vol. 13, no. 2, 1987/88, pp. 314-350.

Department of Mathematics, St. Olaf College, Northfield, Minnesota 55057

E-mail address: humke@stolaf.edu 